\title{
New Simple Procedure for the Computation of the Multimode Admittance or Impedance Matrix of Planar Waveguide Junctions
}

\author{
Alejandro Alvarez Melcon, Graham Connor, and Marco Guglielmi, Member, IEEE
}

\begin{abstract}
Much effort has been devoted in the past to the development of electromagnetic simulation algorithms for waveguide junctions that could at the same time include higher-order mode interactions and lead to computationally efficient codes. Even though this is indeed a classical subject, there is still a strong interest in this area as waveguide systems become increasingly complex, and ever shorter development and manufacturing times are required. In this paper a simple method is described for the evaluation of the multimode network representation of planar waveguide junctions in terms of admittance or impedance parameters. The key feature of the method is that it starts from the wanted final results, the equivalent network representation, in order to obtain an analytic expression for the evaluation of the relevant matrix elements. The procedure is based on general network theory and is equivalent to ideally measuring directly the value of the admittance or impedance elements. In this paper the evaluation procedure is fully described. Measured results of actual hardware are then compared with simulations indicating that the codes developed are indeed very accurate as well as computationally very efficient.
\end{abstract}

\section{INTRODUCTION}

$\mathbf{T}$ HE DESCRIPTION of the microwave properties of arbitrary planar waveguide junctions, like the one shown in Fig. 1, is a subject that has been studied considerably in the past. Of particular interest, from the engineering point of view, have been equivalent network representations, such as the ones that can be found in [1], for instance. The results in [1], however, only account for single mode interactions and are therefore not suitable for the design of modern microwave components. Higher order mode interactions have been traditionally accounted for using mode-matching procedures [2]. The resulting codes, however, although accurate, can be computationally very inefficient.

Recently, a multimode equivalent network representation for arbitrary waveguide junctions has been developed which is based on an integral equation formulation [3]. In [3], the imposition of the boundary conditions leads to an integral equation and to an equivalent network representation of the junction. Solving the integral equation allows for the subsequent computation of the multimode coupling matrix elements. Although this approach can be made computationally efficient, it requires substantial analytical work so that a better approach was deemed necessary.

Manuscript received April 9, 1995; revised November 27, 1995.

The authors are with the European Space Research and Technology Centre (ESTEC), 2200 AG Noordwijk, The Netherlands.

Publisher Item Identifier S 0018-9480(96)01561-X.

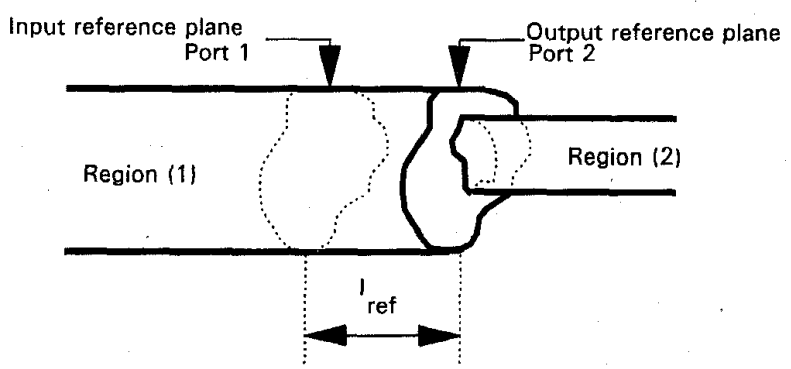

Fig. 1. Arbitrary planar wayeguide junction studied in this paper.

In this paper a simple method is described for the evaluation of the multimode network representation of planar waveguide junctions in terms of admittance parameters or impedance parameters. The key feature of the method is that it starts from the wanted final results, the equivalent network representation, in order to obtain an analytic expression for the evaluation of the relevant matrix elements. The procedure is based on general network theory and is equivalent to ideally measuring directly the value of the admittance elements. The procedure is fully described leading to simple and elegant analytical expressions that are valid for arbitrary planar waveguide junctions. Measurement results of actual hardware are then compared with simulations indicating that the codes developed are indeed very accurate as well as computationally very efficient.

\section{ADMITTANCE FORMULATION}

The structure under investigation is the planar junction between two arbitrary waveguides shown in Fig. 1. The first step of the procedure is to define input and output reference planes (see Fig. 1), and to write formally the wanted final result in the form

$$
I_{m}^{(\delta)}=\sum_{n=1}^{\infty} Y_{m, n}^{(\delta, 1)} \cdot V_{n}^{(1)}+\sum_{n=1}^{\infty} Y_{m, n}^{(\delta, 2)} \cdot V_{n}^{(2)}
$$

Where $I_{m}^{(\delta)}$ and $V_{n}^{(\delta)}$ are modal currents and voltages, respectively, and where $\delta$ can be 1 for region (1) or 2 for region (2), as shown in Fig. 1. The modal currents and voltages in (1) are the amplitude of the vector mode functions $\boldsymbol{h}_{m}^{(\delta)}$ and $\boldsymbol{e}_{m}^{(\delta)}$ relevant to the waveguide cross section $(\delta)$, as defined in [1], for instance. The above system of linear equations 


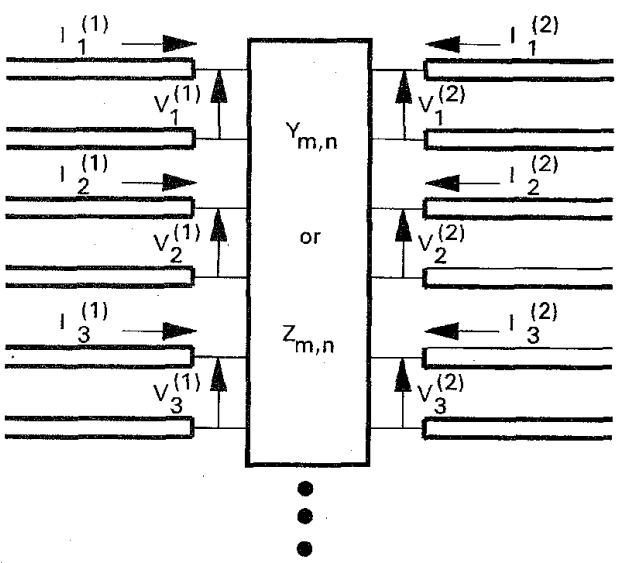

Fig. 2. Multimode equivalent network representation of the junction shown in Fig. 1, in terms of admittance parameters.

is equivalent to the network representation shown in Fig. 2 and can be easily solved with a computer program to study structures involving waveguide junctions. In order to analyze more complex waveguide structures, composed of a number of junctions connected by lengths of uniform waveguide, we only need to define a larger linear system which will have a simple block diagonal coefficient matrix. Each block will in fact represent either a junction or a length of connecting uniform waveguide.

The key point of the approach described in this paper is to note that (1) can also be used to obtain a formal expression for the evaluation of the $Y_{m, n}$ elements. We can, in fact, write directly

$$
Y_{m, n}^{(\delta, \gamma)}=\frac{I_{m}^{(\delta)}}{V_{n}^{(\gamma)}} ; \quad \forall V_{i}^{(\xi)}=0, \quad i \neq n, \xi \neq \gamma .
$$

Where $\gamma$ and $\xi$ can be again 1 for region (1) or 2 for region (2), respectively. This expression is the definition of $Y_{m, n}$ in terms of network theory and can also be used to evaluate directly the actual $Y_{m, n}$ elements, formally yielding

$$
Y_{m, n}^{(\delta, \gamma)}=\frac{\int_{c s(\delta)} \boldsymbol{H}^{(\delta)}\left[V_{n}^{(\gamma)}\right] \cdot \boldsymbol{h}_{m}^{(\delta)} d s^{\prime}}{V_{n}^{(\gamma)}}
$$

where $H^{(\delta)}\left[V_{n}^{(\gamma)}\right]$ is the transverse magnetic field generated at the port $(\delta)$ by the incident mode of amplitude $V_{n}^{(\gamma)}$ in port $(\gamma)$.

To explain in detail the use of (2) and (3), let us begin with $Y_{m, n}^{(1,1)}$. For this elements, the definition in (2) requires a single mode incident from the left (port 1) (which corresponds to $V_{n}^{(1)} \neq 0$ and all other voltages in port 1 equal zero) and a short circuit in port 2 (corresponding to all voltages in region (2) equal zero). The resulting structure becomes the one shown in Fig. 3 and, as we can see, no discontinuities are actually present so that we can write directly

$$
Y_{m, n}^{(1,1)}=(-j) \cdot Y_{0 m}^{(1)} \cdot \cot \left(\beta_{m}^{(1)} l_{r e f}\right) \cdot \delta_{m, n} .
$$

Following the same philosophy for the elements $Y_{m, n}^{(2,1)}$, the definition in (2) requires again a single mode incident from

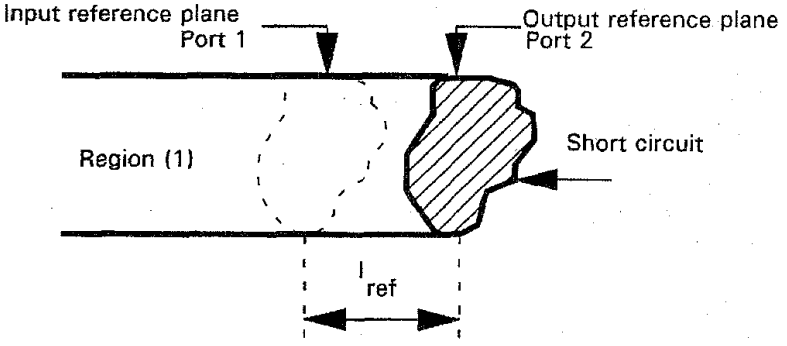

Fig. 3. Structure used for the evaluation of the $Y_{m ; n}^{(1,1)}$ and $Y_{m, n}^{(2,1)}$. A short circuit is placed at port 2 of Fig. 1.

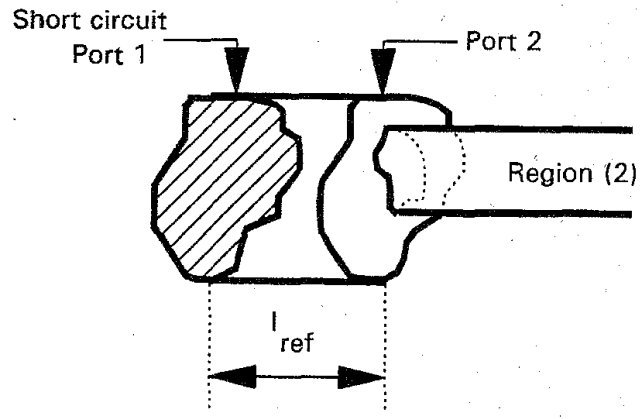

Fig. 4. Structure used for the evaluation of the $Y_{m, n}^{(2,2)}$. A short circuit is placed at port 1 of Fig. 1 .

the left (port 1) and a short circuit in port 2. The current response will now be measured at port 2 using standard orthogonality, and the same structure in Fig. 3. Using then simple transmission line calculations, the resulting expression is .

$$
Y_{m, n}^{(2,1)}=(-j) \cdot \frac{Y_{0 m}^{(1)}}{\sin \left(\beta_{m}^{(1)} l_{\text {ref }}\right)} \cdot\left\langle\boldsymbol{h}_{m}^{(\mathbf{1})} \boldsymbol{h}_{n}^{(2)}\right\rangle
$$

which can also be used for $Y_{n, m}^{(1,2)}$. since the junction is lossless and reciprocal.

Finally, for the elements $Y_{m, n}^{(2,2)}$, the definition in (2) requires a single mode exciting from the right (port 2) and a short circuit at port 1. The situation is therefore as indicated in Fig. 4 , and the current response is again taken in port 2. To use (3), we now need to write an expression for $\boldsymbol{H}^{(2)}$. Taking into account the continuity of the transversal magnetic field at port (2), we can write

$$
H^{(2)}=\sum_{k=1}^{\infty} I_{n}^{(1)} h_{k}^{(1)}
$$

Using again simple transmission line theory, we have

$$
\begin{aligned}
Y_{m, n}^{(2,2)}= & \sum_{k=1}^{\infty}(-j) Y_{0 k}^{(1)} \cdot \cot \left(\beta_{k}^{(1)} l_{r e f}\right) \cdot\left\langle\boldsymbol{e}_{k}^{(1)} \boldsymbol{e}_{n}^{(2)}\right\rangle \\
& \cdot\left\langle\boldsymbol{h}_{k}^{(1)} \boldsymbol{h}_{m}^{(2)}\right\rangle .
\end{aligned}
$$

This concludes the formal derivation of the multimode admittance matrix elements. What is important to note at this 


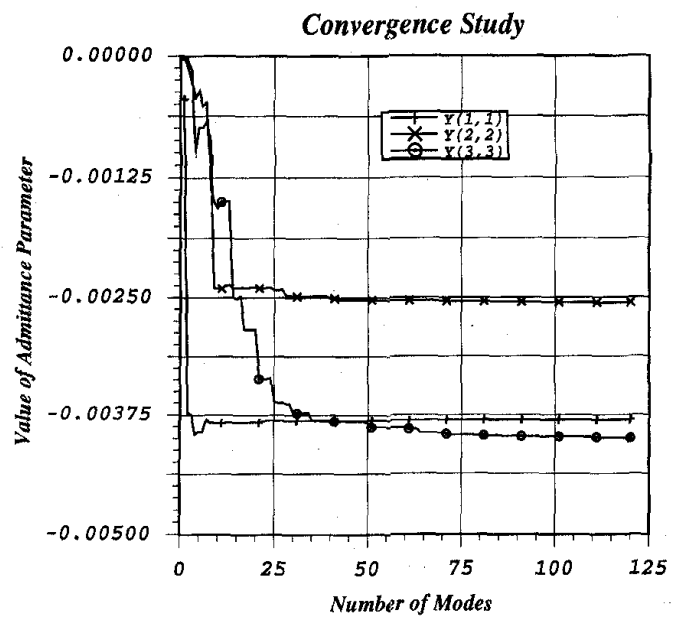

Fig. 5. Typical convergence behavior of the super $(2,2)$ terms. The transition studied is from centered rectangular ( $a=19.05 \mathrm{~mm}, b=9.525 \mathrm{~mm}$ ) to circular $(r=13.6 \mathrm{~mm})$ waveguide.

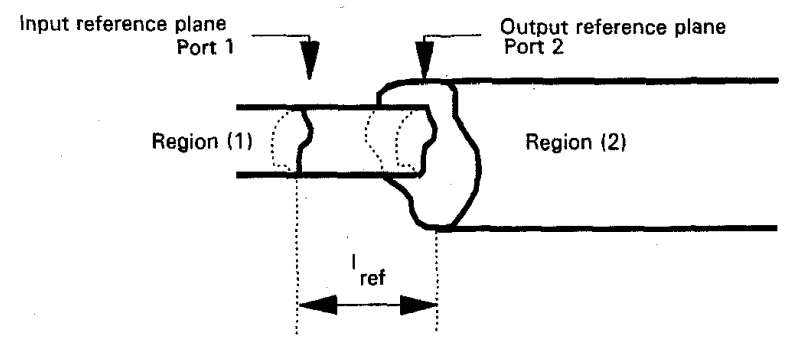

Fig. 6. Position of the reference planes in the case of an impedance representation.

point is the global structure of the admittance matrix that we have obtained. The matrix itself can be subdivided into four blocks. One block contains only single entries in the main diagonal (super $(1,1)$ elements). Two more blocks contain identical single entry elements (super $(1,2)$ and $(2,1)$ ). Only the last block (super $(2,2)$ elements) requires the computation of summations. Furthermore, the running index of the summations affect only the modes of the larger waveguide, namely region (1). A typical example of convergence of the summations is shown in Fig. 5. The results shown in Fig. 5 are for a centered junction between a rectangular waveguide ( $a=19.05 \mathrm{~mm}, b=9.525 \mathrm{~mm}$ ) and a circular waveguide $(r=13.60 \mathrm{~mm})$, the length $l_{\text {ref }}$ is $5 \mathrm{~mm}$, the frequency of operation is $15 \mathrm{Ghz}$. The parameters $Y_{1,1}, Y_{2,2}$, and $Y_{3,3}$ refer to the $\mathrm{TE}_{1,0}, \mathrm{TE}_{3,0}$, and $\mathrm{TE}_{1,2}$ modes, respectively.

For the correct use of the network representation developed, the convergence of the admittance matrix elements must be verified in each case. The value of $l_{\text {ref }}$ used can affect the convergence rate, however, a value of $5 \mathrm{~mm}$ has been found to be suitable for most applications.

In all of the equations derived the symbol \langle\rangle stands for the scalar product of the quantities involved (coupling integrals resulting from the use of the orthogonality conditions) and $\boldsymbol{e}$ and $\boldsymbol{h}$ are the normalized vector mode functions of the

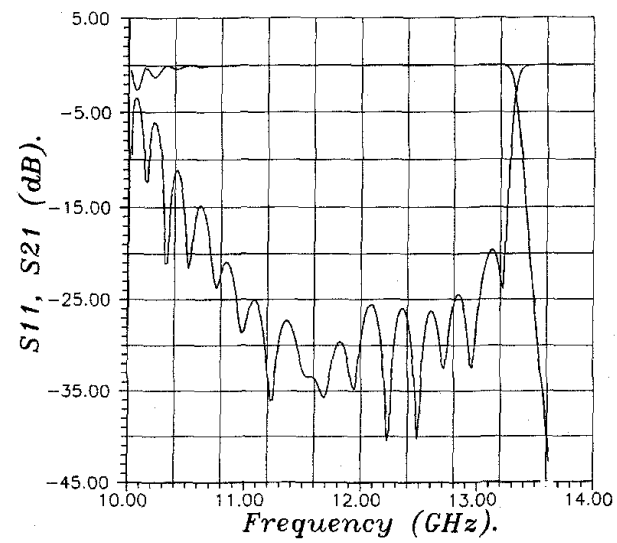

Fig. 7. Simulated results, obtained with the software developed, for the low-pass filter designed by COM DEV Canada. The filter is composed of 85 discontinuities.

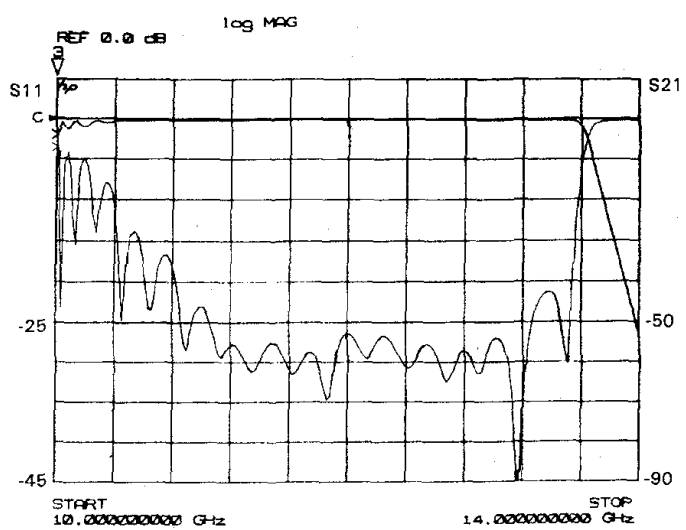

Fig. 8. Measured results of the low-pass filter designed by COM DEV Canada.

waveguides in the appropriate regions. $\beta_{m}^{(1)}$ is the propagation constant of the modes in region (1) and $Y_{0 m}^{(1)}$ is the corresponding characteristic admittance. Moreover $l_{\text {ref }}$ is the separation between the reference planes, as indicated in Fig. 1. The explicit analytic expressions of the scalar products are very simple, depend on the specific waveguide used, and are not reported here for the sake of space.

Admittance matrix descriptions for this type of problems have already been described in the past by several authors and have been identified with different names (see [4] and [5], for instance). A common feature of all these contributions, however, is that they are generally based on the theory of resonators, as described in [6]. As a result, the formulation always requires two type of modes (the so-called $\boldsymbol{e}$ and $\boldsymbol{F}$ modes) and all of the entries of the admittance matrix obtained contain summations over these modes. The method described in this paper is therefore substantially more efficient from a computational point of view. The only contribution (to the authors knowledge) can be found in the technical literature describing an approach similar to the one described here is [7], in the context of rectangular waveguide T-junctions. 


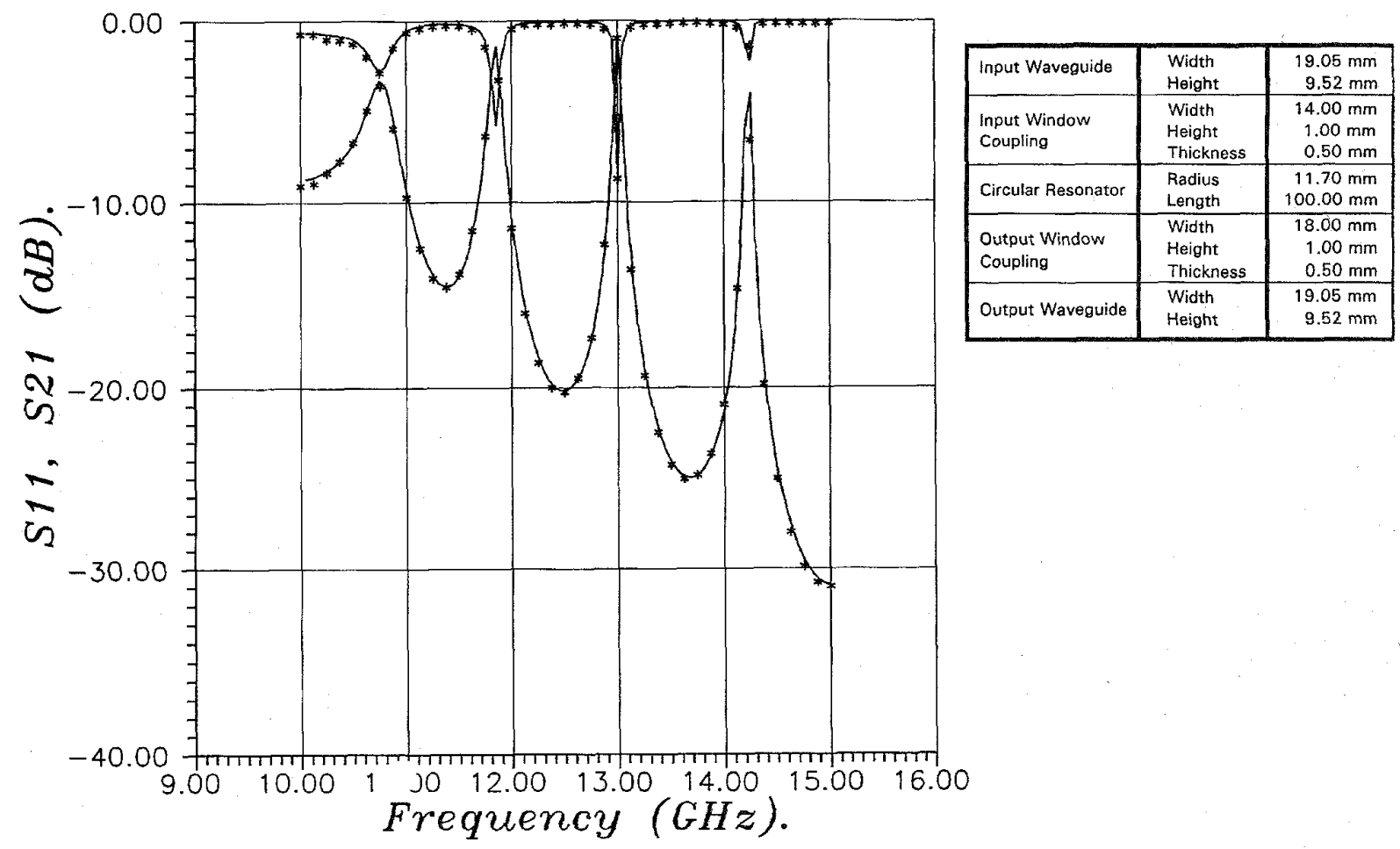

Fig. 9. Comparison between simulated results and measurements for a single circular resonator with different input and output rectangular couplings. The stars are the measured results while the full line represents the results obtained with our approach

\section{IMPEDANCE FORMULATION}

The impedance formulation is essentially complementary to what has already been described for the admittance formulation. The starting point is the following mathematical description of the junction

$$
V_{m}^{(\delta)}=\sum_{n=1}^{\infty} Z_{m, n}^{(\delta, 1)} \cdot I_{n}^{(1)}+\sum_{n=1}^{\infty} Z_{m, n}^{(\delta, 2)} \cdot I_{n}^{(2)} .
$$

The location of the reference planes, however, must be changed to the one shown in Fig. 6, the multimode equivalent network representation obtained is the same as in Fig. 2, with the admittance matrix replaced by an impedance matrix. The formal definition of the generic $Z_{m, n}^{(\delta, \gamma)}$ element is now given by

$$
Z_{m, n}^{(\delta, \gamma)}=\frac{V_{m}^{(\delta)}}{I_{n}^{(\gamma)}} ; \quad \forall I_{i}^{(\xi)}=0, \quad i \neq n, \xi \neq \gamma .
$$

Furthermore, in the derivation of the explicit expressions of the $Z_{m, n}^{(\delta, \gamma)}$ elements, the short-circuit terminations used for the admittance case now become open-circuit terminations, as required by (9). The final expression thus obtained are

$$
\begin{aligned}
& Z_{m, n}^{(1,1)}=(-j) \cdot Z_{0 m}^{(1)} \cdot \cot \left(\beta_{m}^{(1)} l_{\mathrm{ref}}\right) \cdot \delta_{m, n} \\
& Z_{m, n}^{(2,1)}=(-j) \cdot \frac{Z_{0 m}^{(1)}}{\sin \left(\beta_{m}^{(1)} l_{\mathrm{ref}}\right)} \cdot\left\langle\boldsymbol{e}_{n}^{(1)} \boldsymbol{e}_{m}^{(2)}\right\rangle \\
& Z_{m, n}^{(2,2)}=\sum_{k=1}^{\infty}(-j) Z_{0 k}^{(1)} \cdot \cot \left(\beta_{k}^{(1)} l_{\mathrm{ref}}\right) \cdot\left\langle\boldsymbol{h}_{k}^{(1)} h_{n}^{(2)}\right\rangle \cdot\left\langle\boldsymbol{e}_{k}^{(1)} \boldsymbol{e}_{m}^{(2)}\right\rangle
\end{aligned}
$$

\section{CHOOSING THE APPROPRIATE NETWORK REPRESENTATIONS}

Two types of equivalent network representations have been developed, one based on an admittance multimode coupling matrix and the other based on an impedance matrix. The two representations are in principle absolutely equivalent. There is, however, a major difference between them that can be used to choose the more appropriate representation for a given problem. The difference comes from the analytical form of the matrix elements $Y_{m, n}^{(2,2)}$ and $Z_{m, n}^{(2,2)}$. To compute the values of these elements, a summation must be carried out, and the parameters involved are the order of the mode included in the final equivalent network and the index up to which the summation must be carried out. Normally, the number of modes included in the final network ( $m, n$ in (7) and (12)) is always less than 20 , while the number of terms required in the summation ( $k$ in $(7)$ and (12)) needs to be about 150 for good convergence. The summation index $k$, however, only involves the modes of the waveguide region in which the length $l_{\text {ref }}$ is required and not the other.

If the modes of both waveguide regions of the junction have known analytical form, then the admittance or impedance formulation will give the same result with essentially the same computational effort. If one of the two waveguide regions has modes that are defined only numerically (as the result of an eigenvalue problem for an arbitrary waveguide, for instance) then choosing the representation that requires the length $l_{\text {ref }}$ to be in the waveguide region that has the modes specified analytically will result in a much reduced computational effort. 


\section{EXAMPLES OF APPLICATIONS}

As an example of application, the admittance formulation was used to develop a software tool for the analysis of rectangular to rectangular and circular to rectangular waveguide junctions. Since analytical expressions are available for the modes in all regions [1], the scalar products can be easily computed and the admittance elements are then obtained by direct application of the above equations. For the circular to rectangular junction, however, the required scalar products (coupling integrals) have been evaluated numerically. The theory proposed in [8] and [9] is used in this case to convert the surface integrals to line integrals over the contour of the rectangular waveguide, thus increasing the speed of the computation.

Once the admittance coupling matrices are computed, a complex structure, formed by cascading a number of discontinuities, can be easily studied by building a global multimode equivalent network representation. An important feature of this method is that the analysis of the global network thus obtained requires only one inversion per frequency point of a system of linear equations, which is banded and therefore can be performed very efficiently. No intermediate inversions are required for the computation of the $s$-parameters of the complete structure, thus leading to codes that can be very fast even for very complex structures.

The software developed was first used to analyze a lowpass filter composed of 85 discontinuities (capacitive steps) including two double-plane step transformers at the input and the output of the filter. The filter has been designed by COM DEV Canada and manufactured by COM DEV UK. The simulated results obtained with our software are shown in Fig. 7 and can be compared with the measured response of the hardware in Fig. 8. It can be seen that the agreement is very good. The only factor of disagreement is in a frequency shift of about $200 \mathrm{MHz}$ due to the fact that the hardware was manufactured with rounded corners while in the simulation all corners are assumed to be without any radius.

For this analysis 20 modes were used in the final equivalent network and 150 terms were used for the summation of the admittance elements. The analysis was performed on an IBM RISC 6000 platform and the time spent by the software for the analysis of the complete structure was 1 min per each frequency point.

As a further example, the analysis of a circular waveguide resonator with different input and output rectangular-window couplings was performed. In Fig. 9 we present the comparison between simulated results and measurements (the stars) showing a very good agreement. For this analysis four modes were used in all uniform waveguide sections and 120 modes were used to sum the admittance elements. The time spent by the software for the analysis of the complete structure was 2 min and $10 \mathrm{sec}$ for 100 frequency points.

\section{CONCLUSION}

A simple and elegant procedure has been developed for the evaluation of the multimode equivalent network representation of a planar junction between arbitrary waveguides in terms of an admittance or an impedance matrix. The expressions derived can be used for arbitrary waveguides and are particularly simple to use if the modes of at least one of the two waveguides involved are known analytically.

Comparison between the results of simulations obtained with software based on the procedure described and measurements of actual hardware have also been presented indicating that the new procedure indeed leads to very accurate and fast CAD tools.

\section{ACKNOWLEDGMENT}

The authors wish to thank Dr. W. C. Tang and Dr. R. Cameron of COM DEV for supplying the data of the Iow pass filter.

\section{REFERENCES}

[1] N. Marcuvitz, Waveguide Handhook, IEE Electromagnetic Waves Series 21. New York: McGraw-Hill.

[2] H. Patzelt and F. Arndt, "Double-plane steps in rectangular waveguides and their application for transformers, irises, and filters," IEEE Trans. Microwave Theory Tech., vol. MTT-30, no. 5, May 1982.

[3] A. Alvarez and M. Guglielmi, "Multimode network representation of two dimensional steps in rectangular waveguides," in 24th Eur. Microwave Conf., Cannes, France, 1994.

[4] F. Alessandri et al. "Admittance matrix formulation of waveguide discontinuity problems: computer-aided design of branch guide directional couplers," IEEE Trans. Microwave Theory Tech., vol. 36, no. 2, Feb. 1988.

[5] P. Arcioni, M. Bressan, and G. Conciauro, "A new algorithm for the wide-band analysis of arbitrary shaped planar circuits," IEEE Trans. Microwave Theory Tech., vol. 36, no. 10, pp. 1426-1437, Oct. 1988.

[6] K. Kurokawa, An Introduction to the Theory of Microwave Circuits. New York: Academic, 1969.

[7] E. D. Sharp, "An exact calculation for a T-junction of rectangular waveguides having arbitrary cross sections," IEEE Trans. Microwave Theory Tech., vol. MTT-15, no. 2, pp. 109-116, Feb. 1967.

[8] Philippe Guillot et al. "Improvement in Calculation of Some Surface Integrals: Application to Junction Characterization in Cavity Filter Design," IEEE Trans. Microwave Theory Tech., vol. 41, no. 12, Dec. 1993.

[9] G. G. Gentili, "Properties of TE-TM mode-matching techniques," IEEE Trans. Microwave Theory Tech., vol. 39, no. 9, Sept. 1991.

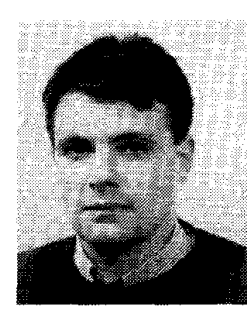

Alejandro Alvarez Melcon was born in Madrid, Spain, in 1965. He received the degree "Ingeniero de Telecomunicaciones" from the Polytechnic University of Madrid (UPM), Spain, in 1991.

In 1988 he joined the Signal, Systems and Radiocommunications Department of UPM as a Research Student, where he was involved in the design, testing, and measurement of broadband spiral antennas for EMS equipment. From 1991 to 1994 he joined the Radio Frequency Systems Division of the European Space Agency in Noordwijk, The Netherlands, where he was involved in the development of analytical and numerical tools for the study of waveguide discontinuities, planar transmission lines, and microwave filters. In 1994 he Joined the Ecole Polytechnique Federale of Lausanne, Lausanne, Switzerland, where he is currently working toward the Ph.D. degree in applied electromagnetism. 


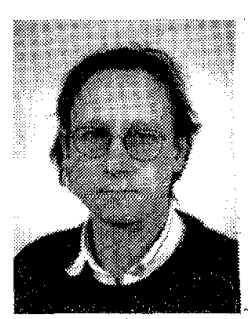

Graham Connor was born in Workington, England, in 1957 . He received the B.Sc. degree in physics from the University of Warwick, Coventry, England, in 1978 .

He joined Marconi Space and Defense Systems, Stanmore, England, where he was responsible for the development of a novel phase equalization structure for a ship-borne antenna. After becoming responsible for theoretical aspects of filter and multiplexer design for the Filter Group, he joined British Aerospace, Stevenage, England, and continued his interest in microwave filter design with the Satellite Payload Department, designing flight filters, output multiplexers, and diplexers for the Filter Group. In 1993 he moved to the RF Systems Division of the European Space Research and Technology Center, Noordwijk, Netherlands, where he continues his interests in the development of microwave filters, multiplexers, passive components, and CAD tools to reduce the design to fight period for flight equipment.

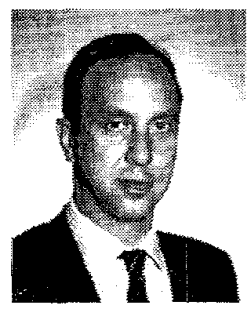

Marco Guglielmi (M'78) was born in Rome, Italy, on December 17, 1954. He received the degree "Laurea in Ingegneria Elettronica" in 1979 from the University of Rome "La Sapienza," Rome, Italy. In 1981 he was awarded. a Fulbright Scholarship in Rome, Italy, and an HISP scholarship (Halsey International Scholarship Program) from the University of Bridgeport, Bridgeport, $\mathrm{CT}$, where in 1982 he received the M.S. degree in electrical engineering. In 1986 he received the $\mathrm{Ph} . \mathrm{D}$. degree in electrophysics from Polytechnic University, Brooklyn, NY.

From 1984 to 1986 he was Academic Associate at Polytechnic University, and from 1986 to 1988 he was Assistant Professor at the same institution. From 1988 to 1989 he was Assistant Professor at the New Jersey Institute of Technology, Newark, NJ. In 1989 he joined the RF System Division of the European Space Research and Technology Centre, Noordwijk, Netherlands, where he is currently involved in the development of passive microwave components for space applications. His professional interests include the areas of solid-state devices and circuits, periodic structures, phased arrays and millimeter-wave leaky-wave antennas, network representations of waveguide discontinuities, and microwave filtering structures. 